\title{
The Impact of Therapeutic Hypothermia Used to Treat Anoxic Brain Injury After Cardiopulmonary Resuscitation on Organ Donation Outcomes
}

\author{
Charles Wright, MD, ${ }^{1, *}$ Madhukar S. Patel, MD, MBA, ScM, ${ }^{2, *}$ Xiang Gao, MD, MPH, ${ }^{3}$ \\ Maxwell Witt, BS, ${ }^{4}$ Mitchell Sally, MD, FACS, ${ }^{5,6}$ Tahnee Groat, MPH, ${ }^{5}$ Megan Crutchfield, MPH, \\ Nikole Neidlinger, MD, FACS, ${ }^{7,8}$ Markeith Pilot, MPH, ${ }^{9}$ Darren J. Malinoski, MD, FACS ${ }^{5,6}$; \\ and the Organ Donation Research Consortium (ODRC) Anoxic Organ Donor Study Group
}

Therapeutic hypothermia (TH) is clinically used to improve neurologic outcomes in patients with anoxic brain injury after cardiopulmonary resuscitation (CPR). For patients that regress and become organ donors after neurologic determination of death (DNDDs), the impact of TH received before determination of death on organ donation outcomes remains unknown. A prospective observational study of all adult DNDDs that received CPR and had anoxia as a cause of death from March 2013 to December 2014 was conducted across 20 organ procurement organizations (OPOs) in the United States. Main outcome measures included organs transplanted per donor (OTPD), specific organ transplantation rates, and recipient graft outcomes. One thousand ninety eight DNDDs met inclusion criteria, with $46 \%$ having received TH before determination of death. DNDDs with hypothermia before death had a similar number of OTPD $(2.74$ vs. $2.69, p=0.61)$ and similar transplantation rates of individual organs. With regards to recipients, there was significantly less delayed graft function (DGF) in kidney grafts from donors who received TH before death (24\% vs. 30\%, $p=0.02)$. After adjusting for donor, recipient, and graft related factors, the protective effect of TH on DGF persisted (OR 0.75, 95\% CI [0.56-0.995], $p=0.046)$. TH before death in the donor is independently associated with a $25 \%$ decrease in DGF among kidney recipients. This should be considered a protective donor selection factor in guiding the decision to accept or reject an organ for transplantation.

Keywords: hypothermia, deceased donation, cardiopulmonary resuscitation, delayed graft function

\section{Introduction}

T HE INDUCTION OF mild therapeutic hypothermia (TH) has been found to improve outcomes after anoxic brain injury in adults with out-of-hospital cardiac arrest (Bernard et al., 2002; Hypothermia after Cardiac Arrest Study, 2002). As such, after anoxic brain injury, some patients will improve and survive. Others, however, may regress with eventual brainstem herniation and have the potential to become organ donors after neurologic determination of death (DNDDs). For those patients who neither survive nor regress to brainstem herniation and whose family withdraws life-sustaining measures, donation after circulatory determination of death (DCDD) may be a possibility.

Recently, a large prospective, randomized controlled trial assessed the impact of TH in organ donors after determination 2017.

${ }^{1}$ LifeLink of Florida, Tampa, Florida.

${ }^{2}$ Department of Surgery, Massachusetts General Hospital, Boston, Massachusetts.

${ }^{3}$ Department of Surgery, University of Iowa, Iowa City, Iowa.

${ }^{4}$ School of Medicine, University of Michigan, Ann Arbor, Michigan.

${ }^{5}$ Surgical Critical Care Section, Portland Veterans Affairs Medical Center, Portland, Oregon.

${ }^{6}$ Department of Surgery, Oregon Health \& Science University, Portland, Oregon.

${ }^{7}$ Donor Network West, San Ramon, California.

${ }^{8}$ California Pacific Medical Center, San Francisco, California.

${ }^{9}$ OneLegacy, Los Angeles, California.

*These authors are co-first authors and contributed equally to this work. 
of death by neurologic criteria (Niemann and Malinoski, 2015). Specifically, this study found that in transplant recipients receiving renal allografts from donors treated with mild hypothermia $\left(34^{\circ} \mathrm{C}\right.$ to $\left.35^{\circ} \mathrm{C}\right)$, the relative odds of delayed graft function (DGF) was 38\% lower than those receiving kidneys from donors maintained at normothermia $\left(36.5^{\circ} \mathrm{C}\right.$ to $37.5^{\circ} \mathrm{C}$ ) during the donor management process (Niemann and Malinoski, 2015). Furthermore, greater relative benefit was noted in recipients of organs from expanded-criteria donors, suggesting that the subsequent ischemia-reperfusion injury in recipients of these higher risk organs can potentially be ameliorated with mild hypothermia (Niemann and Malinoski, 2015).

Currently, the impact that $\mathrm{TH}$ before death has on organ donation outcomes remains unknown. Given this, and the known clinical use of TH in patients with anoxic brain injury after cardiopulmonary resuscitation (CPR), we sought to investigate whether $\mathrm{TH}$ before determination of death impacts organ donation outcomes.

\section{Materials and Methods}

\section{Study design}

Twenty organ procurement organizations (OPOs) across the United States (US; see Acknowledgments section for list of participating organizations) prospectively collected data on all adult ( $\geq 18$ years old) DNDDs who received CPR and were noted to have anoxia as a cause of death (Fig. 1). Data were collected from March 2013 to December 2014. Stan- dard criteria donors (SCDs), as well as expanded criteria donors (ECDs), were included, whereas DCDDs were excluded. Furthermore, this study used data from the Organ Procurement and Transplantation Network (OPTN), which includes data on all donors, wait-listed candidates, and transplant recipients in the US and has been described elsewhere (Leppke et al., 2013). The Health Resources and Services Administration (HRSA), US Department of Health and Human Services provides oversight to the activities of the OPTN. Finally, this study was determined to be nonhuman subjects' research by the Portland VA Medical Center (PVAMC) Research and Development Committee, and each OPO obtained approval from their local research oversight entity. All DNDDs were managed per OPO-specific donor management protocols based on the United Network for Organ Sharing (UNOS) clinical pathway.

\section{Data collection and outcome measures}

Data were collected by each OPO using a standardized electronic form that was completed by OPO personnel at the bedside of each patient during donor management or through medical chart review while assessing suitability of potential organ donors. Data obtained included donor demographics, treatments received before neurologic determination of death (TH and CPR), donor type (SCD or ECD), and organs transplanted (heart, lung, kidney, liver, intestine, and/or pancreas). Completed data forms were sent to the coordinating center (VAPORHCS) upon completion. To assess

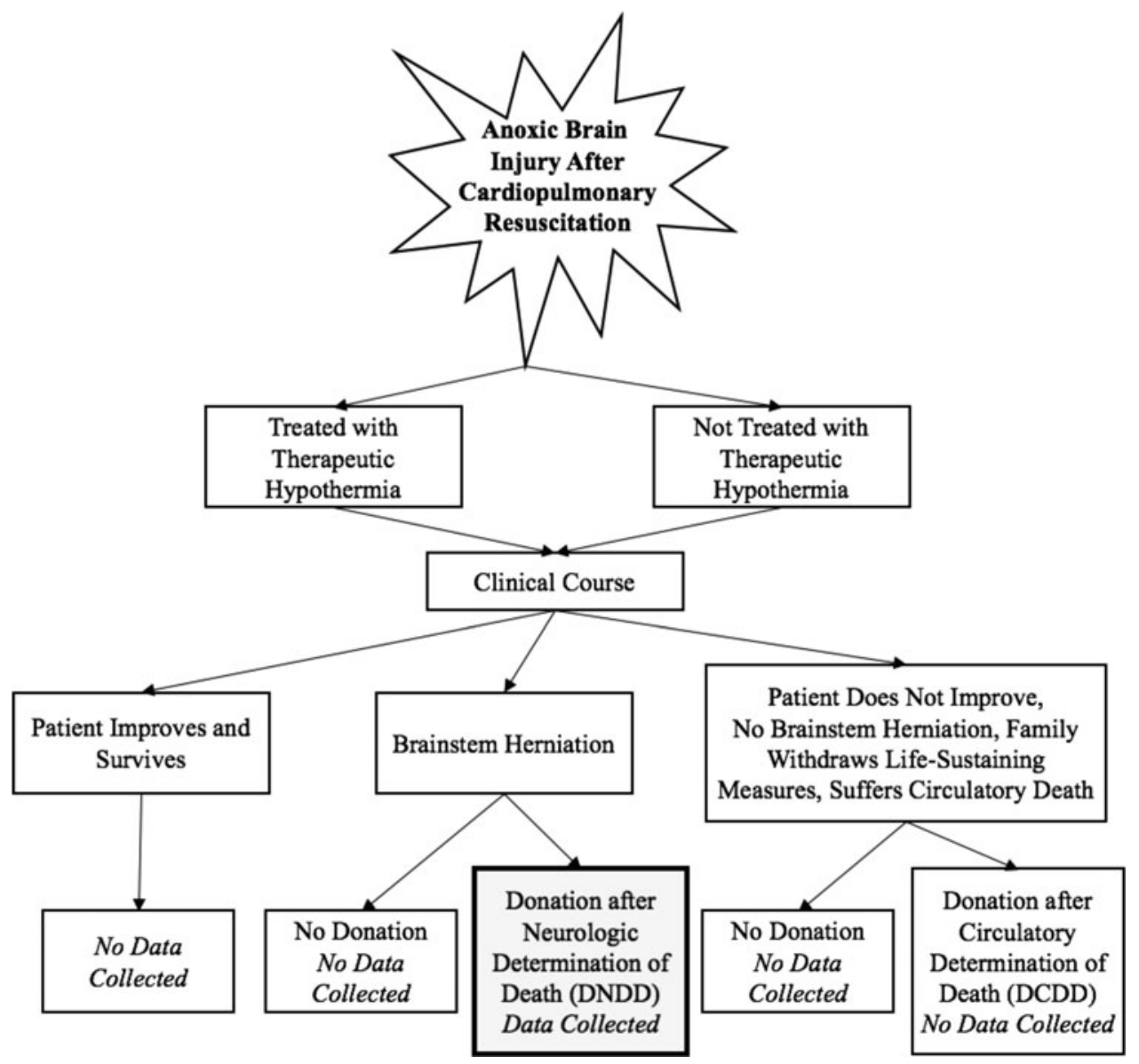

FIG. 1. Clinical criteria for including donors after neurologic determination of death. 
Table 1. Demographics of Donors After Neurologic Determination of Death

\begin{tabular}{lccc}
\hline Variable & $\begin{array}{c}\text { All DNDDs } \\
(\mathrm{n}=1098)\end{array}$ & $\begin{array}{c}\text { Without hypothermia } \\
\text { before death } \\
(\mathrm{n}=588)\end{array}$ & $\begin{array}{c}\text { With hypothermia } \\
\text { before death } \\
(\mathrm{n}=510)\end{array}$ \\
\hline Age (years) & $41.7 \pm 14.4$ & $41.6 \pm 14.6$ & $41.8 \pm 14.2$ \\
Gender (female) & $519(47 \%)$ & $288(49 \%)$ & $231(45 \%)$ \\
Ethnicity & $798(73 \%)$ & $423(72 \%)$ & $375(74 \%)$ \\
White & $143(13 \%)$ & $88(15 \%)$ & $55(11 \%)$ \\
Black & $119(11 \%)$ & $62(11 \%)$ & $57(11 \%)$ \\
Hispanic & $38(4 \%)$ & $15(3 \%)$ & $23(5 \%)$ \\
Other & & & $413(81 \%)$ \\
Donor Type & $892(81 \%)$ & $479(82 \%)$ & $97(19 \%)$ \\
SCD & $206(19 \%)$ & $109(19 \%)$ & 0.81 \\
ECD & & & 0.08 \\
\hline
\end{tabular}

CPR, cardiopulmonary resuscitation; DNDD, donor after neurologic determination of death; SCD, standard criteria donor; ECD, expanded criteria donor.

graft outcomes, linkage to the OPTN database was performed. Main outcome measures included the following: overall number of organs transplanted per donor (OTPD), the proportion of donors with $\geq 4$ OTPD (as this represents a higher average OTPD goal compared to the national goal of 3.75 OTPD established by the Donation and Transplantation Community of Practice [http://healthcarecommunities.org/]), individual organ transplantation rates, and recipient graft outcomes (for heart, lung, kidney, and liver allografts). Regarding transplant recipient graft outcomes, follow-up of graft status was assessed at 1 year through OPTN data and was categorized as either functioning or nonfunctioning based on reported graft failure or recipient death (and the date of such failure/death if failed/deceased) or lack thereof. For kidney grafts, DGF was also assessed based on reported OPTN data and given clinically applicability, as well as simplicity, was defined as the need for dialysis within the first week after transplantation (Mallon et al., 2013).

\section{Statistical analysis}

Differences in donor characteristics, OTPD, organ transplantation rates, and graft outcomes between donors receiving $\mathrm{TH}$ after CPR and before determination of death were compared to those not receiving TH using Pearson's $\chi^{2}$ and analysis of variance (ANOVA) for categorical data and the independent $t$-test for continuous data. For graft outcomes noted to have a $p<0.2$ on univariate analysis, a multivariable analysis was performed. Specifically, this adjusted analysis aimed to assess if $\mathrm{TH}$ remained an independent predictor of graft outcome when the donor, recipient, and graft-related covariates that are utilized in the Scientific Registry of Transplant Recipients (SRTR) risk adjustment models were included in the analysis (http://tools.srtr.org/PSRModelVis App). Variables with a $p<0.05$ on multivariable analysis were felt to be independent predictors. Statistical analysis was performed using SPSS versions 22.0 and 23.0 (SPSS, Armonk, NY: IBM Corporation).

\section{Results}

Over the 22-month study period, 1098 DNDDs met inclusion criteria with $46 \%$ (510) having received $\mathrm{TH}$ before neurologic determination of death. The average overall age was $41.7 \pm 14.4$ years, with $47 \%$ (519) being female and the majority $(81 \%)$ being SCDs. Donor characteristics between treatment groups were similar with regards to age, gender, ethnicity, and donor type (Table 1). In DNDDs receiving hypothermia before brainstem herniation, the average duration of hypothermia was $31.9 \pm 14$ hours.

With regards to organ transplantation rates, there were a similar number of heart, lung, kidney, liver, and pancreas transplanted from DNDDs across treatment groups (Table 2). Intestines, however, were more often transplanted from DNDDs who received hypothermia before brainstem herniation ( $8 \%$ vs. $5 \%, p=0.03$ ). Overall, the mean OTPD for all DNDDs was $2.71 \pm 1.77$, with $31 \%$ (336) having $\geq 4$ OTPD. There was no significant difference across treatment groups with regards to these outcome measures (Table 2).

Upon analysis of graft outcomes, 1-year graft survival for heart, lung, kidney, and liver allografts was noted to be $91 \%$, $86 \%, 95 \%$, and $92 \%$, respectively. These rates were noted to be similar on comparison across treatment groups (Table 3). For kidney transplant recipients, the overall occurrence of DGF was found to be $28 \%$ for the 1211 grafts analyzed. Furthermore, there was significantly less DGF in kidney grafts from donors who received $\mathrm{TH}$ to treat their primary neurologic injury before death $(24 \%$ vs. $30 \%, p=0.02$,

Table 2. Organ Transplantation Rates of Donors After Neurologic Determination of Death

\begin{tabular}{lccc}
\hline & $\begin{array}{c}\text { Without } \\
\text { hypothermia } \\
\text { before } \\
\text { death } \\
(\mathrm{n}=588)\end{array}$ & $\begin{array}{c}\text { With } \\
\text { hypothermia } \\
\text { before } \\
\text { death } \\
(\mathrm{n}=510)\end{array}$ & p-value \\
Variable & & & \\
\hline Transplantation rates & $146(25 \%)$ & $143(28 \%)$ & 0.23 \\
Heart $(n=289)$ & $112(19 \%)$ & $95(19 \%)$ & 0.86 \\
Any lung $(n=207)$ & $383(65 \%)$ & $343(67 \%)$ & 0.46 \\
Any kidney $(n=726)$ & $422(72 \%)$ & $366(72 \%)$ & 0.99 \\
Liver $(n=788)$ & $27(5 \%)$ & $39(8 \%)$ & 0.03 \\
Intestine $(n=66)$ & $75(13 \%)$ & $69(14 \%)$ & 0.71 \\
Pancreas $(n=144)$ & $172(29 \%)$ & $164(32 \%)$ & 0.30 \\
$\geq 4$ OTPD & $2.69 \pm 1.81$ & $2.74 \pm 1.72$ & 0.61 \\
Mean OTPD & &
\end{tabular}

OTPD, organs transplanted per donor. 
Table 3. Graft Outcomes of Donors After Neurologic Determination of Death

\begin{tabular}{lccr}
\hline Variable & $\begin{array}{c}\text { Without hypothermia } \\
\text { before death }(\mathrm{n}=588)\end{array}$ & $\begin{array}{c}\text { With hypothermia } \\
\text { before death }(\mathrm{n}=510)\end{array}$ & p-value \\
\hline 1-year graft outcomes* & & & 0.72 \\
Heart graft survival $(n=289)$ & $90 \%$ & $92 \%$ & 0.41 \\
Lung graft survival $(n=64)$ & $83 \%$ & $91 \%$ & 0.74 \\
Kidney graft survival $(n=1211)$ & $96 \%$ & $95 \%$ & 0.62 \\
Liver graft survival $(n=754)$ & $92 \%$ & $91 \%$ & 0.02 \\
Delayed graft function & & & $24 \%$ \\
$\quad$ Kidney graft $(n=1211)$ & $30 \%$ & & \\
\hline
\end{tabular}

*Only recipients with complete data included.

Table 3). After adjusting for known donor, recipient, and graft-related factors, the protective effect of hypothermia on DGF persisted (OR 0.75, 95\% CI [0.56-0.995], $p=0.046$, Table 4). Of note, the number of kidney grafts pumped by the OPO or transplant center was similar between the hypothermia and nonhypothermia groups ( $42 \%$ vs. $44 \%, p=0.38$, respectively).

\section{Discussion}

In the United States, DNDDs that receive CPR account for greater than 1000 organs transplanted per year (Orioles $\mathrm{et}$ al., 2013). Despite having a lower number of OTPD compared to non-CPR donors (Orioles et al., 2013), recent reports suggest that there are no differences in immediate or long-term graft function in organs from donors who received CPR (Orioles et al., 2013; West et al., 2016). This study explored the previously unknown effects of $\mathrm{TH}$ before determination of death by neurologic criteria in patients with anoxic brain injury after CPR. Although TH before brainstem herniation did not impact overall or individual organ transplantation rates, a statistically significant and clinically relevant protective effect was noted as kidney transplant recipients were found to have a $25 \%$ decrease in DGF after adjusting for donor, recipient, and graft-related factors.

$\mathrm{TH}$, or a controlled reduction in core body temperature, has been used clinically to ameliorate secondary organ damage following a variety of primary insults (Moore et al., 2011). Although the most substantial evidence to date on the beneficial effects of TH has been in neuroprotection, theoretical advantages to other organs, such as the heart and kidney, have been suggested given the systemic effect of hypothermia (Moore et al., 2011; Polderman, 2008). Many potential mechanisms have been implicated in explaining the protective effects of $\mathrm{TH}$, including reduction in metabolic rate; alteration in electrolyte shifts; decreased apoptosis and mitochondrial dysfunction; reduction in ischemia-induced inflammatory and immune reactions; decreased release of free radicals; reduced toxic metabolite accumulation and acidosis; and increased early protective gene activation (Moore et al., 2011; Polderman, 2008, 2009; Yenari and Han, 2012). These mechanisms may be conferred in patients receiving TH for anoxic brain injury after CPR and, thus, provide a potential explanation for the finding that $\mathrm{TH}$ before determination of death by neurologic criteria leads to decreased DGF in kidney transplant recipients. It should be noted that despite TH, similar 1-year graft outcomes for heart, lung, kidney, and liver transplant recipients across exposure groups were noted. This suggests that, at least in the short term, TH in the potential organ donor does not seem to negatively impact graft survival.

The rate of DGF after kidney transplantation has been noted to vary substantially in the literature, likely due to the heterogeneity of diagnostic criteria and thresholds used in its

Table 4. Multivariable Analysis: Independent Predictors of Delayed Graft Function IN KIDNEY TRANSPLANT RECIPIENTS

\begin{tabular}{lccc}
\hline Variable & Odds ratio & 95\% confidence interval for odds ratio & $\mathrm{p}$-value \\
\hline Therapeutic Hypothermia Before Death & 0.75 & $0.56-0.995$ & 0.046 \\
Donor - Age (per year) & 1.02 & $1.004-1.03$ & 0.008 \\
Donor - Weight (per kg) & 0.998 & $0.99-1.004$ & 0.48 \\
Donor - Hypertension & 1.20 & $0.85-1.70$ & 0.30 \\
Donor - Terminal Serum Creatinine & 1.67 & $1.50-1.86$ & $<0.001$ \\
Recipient - Age (per year) & 0.995 & $0.98-1.01$ & 0.410 \\
Recipient - Gender (Male) & 1.83 & $1.34-2.51$ & $<0.001$ \\
Recipient - BMI (per point) & 1.02 & $0.998-1.04$ & 0.08 \\
Recipient - Diabetes & 1.41 & $1.03-1.92$ & 0.03 \\
Recipient - Dialysis Time Before Transplant (per year) & 1.10 & $1.06-1.15$ & $<0.001$ \\
Recipient - Pretransplant Transfusion & 1.25 & $0.88-1.76$ & 0.22 \\
Recipient - Calculated Panel Reactive Antibodies & 1.002 & $0.998-1.01$ & 0.343 \\
Donor and Recipient-HLA mismatch & 1.07 & $0.97-1.19$ & 0.16 \\
Graft - Cold Ischemia Time & 1.03 & $1.02-1.05$ & $<0.001$ \\
Graft - Pump Utilized & 0.68 & $0.50-0.93$ & 0.01 \\
\hline
\end{tabular}

HLA, human leukocyte antigen. 
definition, as well as differences in donor and recipient characteristics across different studies (Mallon et al., 2013). As previously mentioned, in this study, dialysis in the first postoperative week was used given its simplicity and clinical applicability (Mallon et al., 2013). Using this definition, a previous large registry study of deceased donor recipients approximated DGF to occur in $25-28 \%$ of patients (Huaman et al., 2016), a rate that is similar to that found in the current investigation. Clinically, DGF is relevant as it has been associated with negative recipient outcomes (Butala et al., 2013; Fonseca et al., 2015; McLaren et al., 1999; Tapiawala et al., 2010; Yarlagadda et al., 2009). First and foremost, DGF increases resource consumption, total cost of care, and emotional distress (Buchanan et al., 2008; Jordan et al., 2018; Mannon, 2018; Serrano et al., 2018; Zens et al., 2018). In addition, results of a large systematic review and metaanalysis of 33 studies have suggested that DGF is associated with a $38 \%$ higher risk of acute rejection and $41 \%$ increased risk of graft loss compared to recipients without DGF at 3.2 years of follow-up (Yarlagadda et al., 2009). In the current study, hypothermia was not noted to have a protective effect on graft loss.

Given the significant negative consequence of DGF in kidney transplant recipients and the overall incidence of its occurrence, attempts to prevent DGF through donor management have been investigated. Specific strategies include adopting an approach of standardized care of potential organ donors to meet preset donor management goals (Malinoski et al., 2013), the use of low-dose dopamine infusion in the donor (Schnuelle et al., 2009), and avoidance of hydroxyethyl starch during donor management (Patel et al., 2015). In addition, as mentioned above, a prospective, randomized controlled trial noted a significantly decreased rate of DGF in donors that had been preconditioned with mild hypothermia $\left(34^{\circ} \mathrm{C}\right.$ to $\left.35^{\circ} \mathrm{C}\right)$ after declaration of death and during donor management compared to those that were maintained at normothermia $\left(36.5^{\circ} \mathrm{C}\right.$ to $37.5^{\circ} \mathrm{C} ; 28 \%$ vs. $\left.39 \%, p=0.02\right)$ (Niemann et al., 2015). Furthermore, with regard to donor graft preservation, studies comparing hypothermic machine perfusion to simple cold storage have found that the former leads to a significant reduction in DGF for both SCD and more marginal ECD grafts (Gill et al., 2014; Jiao et al., 2013; Moers et al., 2009). Although the aforementioned strategies have been shown to have significant impact in reducing DGF, universal adoption and implementation is challenged due to the need for change in donor management protocols across multiple sites/regions and the need for alignment of multiple providers in the care spectrum (intensivist, OPOs, transplant surgeons, etc.).

This current investigation is the first to evaluate the impact of TH that occurs before neurologic determination of death on donation outcomes. The significant reduction noted in DGF in kidney transplant recipients suggests that the use of $\mathrm{TH}$ in potential donors should be considered as a criterion in risk-adjusted models that help guide the utilization of kidney grafts. Specifically, a refined model for predicting DGF after renal transplantation has been created on the basis of multivariable regression analysis (an associated web-based calculator is available at www.transplantcalculator.com/DGF) (Irish et al., 2010). Using this model, a greater than 50\% DGF risk has been associated with a twofold increase in graft failure (Irish et al., 2010). Donor related factors include ter- minal creatinine, age, weight, donor type, history of hypertension, and cause of death. Furthermore, graft related factors include total number of human leukocyte antigen mismatches, cold and warm ischemia time, and the use of machine perfusion. Given the notable protective effect of $\mathrm{TH}$ before determination of death on DGF in the current study, inclusion of this variable in future models should be considered.

This study is limited by the availability of additional donor and recipient data that could potentially help further discern notable findings. First, although included DNDDs received CPR before brainstem herniation, it is unknown whether the CPR was secondary to out-of-hospital or in-hospital cardiac arrest. In addition, details on the length of CPR time and number of CPR events were not recorded. Second, hypothermia was actively targeted by each donor hospital's critical care team while the donors were still alive and efforts were being made to save them. Local hospital protocols were followed, and the OPOs were not involved in that phase of care. As such, specific details regarding mean temperature and duration of cooling were not recorded by the OPOs. Finally, although there were no negative outcomes noted by $\mathrm{TH}$ on follow-up of recipients, the occurrence of specific side effects, including electrolyte disturbances, hyperglycemia, and potential for infection, would be interesting to note and associate with graft utilization and outcomes.

\section{Conclusion}

The use of TH before determination of death in patients with anoxic brain injury after CPR is independently associated with decreased DGF in kidney transplant recipients. This finding corroborates prior evidence from a randomized control trial that evaluated the impact of $\mathrm{TH}$ after determination of death and authorization for organ donation (Niemann and Malinoski, 2015). Given the potential long-term consequences of DGF after renal transplantation, consideration should be given to incorporating exposure to $\mathrm{TH}$ as a protective donor selection factor in prediction models which evaluate for DGF, as well as other graft outcomes, and thus guide the decision to accept or reject an organ for transplantation.

\section{Authorship Contributions}

Study conception and design: Wright, Patel, Gao, Witt, Crutchfield, Neidlinger, and Malinoski

Acquisition of data: Wright, Gao, Witt, Groat, Crutchfield, Neidlinger, Pilot, and Malinoski

Analysis and interpretation of data: Wright, Patel, Gao, Witt, Sally, and Malinoski

Drafting of article: Wright, Patel, Gao, and Malinoski

Critical revision: Wright, Patel, Witt, Sally, Groat, Crutchfield, Neidlinger, Pilot, and Malinoski

\section{Acknowledgments}

The authors thank and acknowledge the members of the Organ Donation Research Consortium (ODRC) Anoxic Organ Donor Study Group, as well as the donors, recipients, staff, and leadership of the 20 organ procurement organizations, for their support of this study and assistance in collection, as well as management, of data: Bill Tower (Donor 
Network of Arizona); Nikole Neidlinger (Donor Network West); Rick Ash, Scott Bunting, Melissa LeGree, Markeith Pilot (OneLegacy); Jeffrey Trageser (Lifesharing); Matthew Wadsworth (Indiana Donor Network); Dana Green (Louisiana Organ Procurement Agency); Charles Wright (LifeLink of Florida); Shri Bharadwaj (LifeLink of Georgia); Bill Payne (Life Source Upper Midwest Organ Procurement); Emily Stahlschmidt (Mid-America Transplant Services); Jami Gleason (Midwest Transplant Network); Donna Croezen (Carolina Donor Services); Briana Torrey (New England Organ Bank); Kristin Deli Carpini (LiveOnNY); Dan Lebovitz (Lifebanc); Dorrie Dils and Mindy Zoll (Lifeline of Ohio); Chad Muse (LifeShare Transplant Donor Services of Oklahoma); David McCloskey (Pacific Northwest Transplant Bank); Ben Keebler (Southwest Transplant Alliance); and Greg Russell (Intermountain Donor Services).

This research was supported by a grant from the Organ Donor Research Consortium. Furthermore, this research was supported, in part, by an appointment to the Postgraduate Research Participation Program at the Division of Transplantation, U.S. Health Resources and Services Administration (HRSA), U.S. Department of Health and Human Services administered by the Oak Ridge Institute for Science and Education (ORISE) through an interagency agreement between the U.S. Department of Energy and HRSA.

\section{Disclaimer}

Although the data reported here have, in part, been supplied by HRSA, the interpretation and reporting of these data are the responsibility of the author(s) and in no way should be seen as an official policy of or interpretation by the U.S. Government.

\section{Author Disclosure Statement}

Dr. Malinoski received consulting fees and travel reimbursement from Symic.

\section{References}

Bernard SA, Gray TW, Buist MD, et al. Treatment of comatose survivors of out-of-hospital cardiac arrest with induced hypothermia. N Engl J Med 2002;346:557-563.

Buchanan PM, Lentine KL, Burroughs TE, et al. Association of lower costs of pulsatile machine perfusion in renal transplantation from expanded criteria donors. Am J Transplant 2008;8:2391-2401.

Butala NM, Reese PP, Doshi MD, et al. Is delayed graft function causally associated with long-term outcomes after kidney transplantation? Instrumental variable analysis. Transplantation 2013;95:1008-1014.

Fonseca I, Teixeira L, Malheiro J, et al. The effect of delayed graft function on graft and patient survival in kidney transplantation: an approach using competing events analysis. Transpl Int 2015;28:738-750.

Gill J, Dong J, Eng M, et al. Pulsatile perfusion reduces the risk of delayed graft function in deceased donor kidney transplants, irrespective of donor type and cold ischemic time. Transplantation 2014;97:668-674.

Huaman MA, Vilchez V, Mei X, et al. Donor positive blood culture is associated with delayed graft function in kidney transplant recipients: a propensity score analysis of the UNOS database. Clin Transplant 2016;30:415-420.
Hypothermia after Cardiac Arrest Study, G. Mild therapeutic hypothermia to improve the neurologic outcome after cardiac arrest. N Engl J Med 2002;346:549-556.

Irish WD, Ilsley JN, Schnitzler MA, et al. A risk prediction model for delayed graft function in the current era of deceased donor renal transplantation. Am J Transplant 2010;10: 2279-2286.

Jiao B, Liu S, Liu H, et al. Hypothermic machine perfusion reduces delayed graft function and improves one-year graft survival of kidneys from expanded criteria donors: a metaanalysis. PLoS One 2013;8:e81826.

Jordan SC, Choi J, Aubert O, et al. A phase I/II, double-blind, placebo-controlled study assessing safety and efficacy of $\mathrm{C} 1$ esterase inhibitor for prevention of delayed graft function in deceased donor kidney transplant recipients. Am J Transplant 2018;18:2955-2964.

Leppke S, Leighton T, Zaun D, et al. Scientific Registry of Transplant Recipients: collecting, analyzing, and reporting data on transplantation in the United States. Transplant Rev (Orlando) 2013;27:50-56.

Malinoski DJ, Patel MS, Ahmed O, et al. The impact of meeting donor management goals on the development of delayed graft function in kidney transplant recipients. Am J Transplant 2013;13:993-1000.

Mallon DH, Summers DM, Bradley JA, et al. Defining delayed graft function after renal transplantation: simplest is best. Transplantation 2013;96:885-889.

Mannon RB. Delayed Graft Function: the AKI of Kidney Transplantation. Nephron 2018;1-5.

McLaren AJ, Jassem W, Gray DW, et al. Delayed graft function: risk factors and the relative effects of early function and acute rejection on long-term survival in cadaveric renal transplantation. Clin Transplant 1999;13:266-272.

Moers, C., Smits JM, Maathuis MH, et al. Machine perfusion or cold storage in deceased-donor kidney transplantation. N Engl J Med 2009;360:7-19.

Moore EM, Nichol AD, Bernard SA, et al. Therapeutic hypothermia: benefits, mechanisms and potential clinical applications in neurological, cardiac and kidney injury. Injury 2011;42:843-854.

Niemann CU, Feiner J, Swain S, et al. Therapeutic Hypothermia in Deceased Organ Donors and Kidney-Graft Function. N Engl J Med 2015;373:405-414.

Niemann CU, Malinoski D. Therapeutic Hypothermia in Deceased Organ Donors and Kidney-Graft Function. N Engl J Med 2015;373:2687.

Orioles A, Morrison WE, Rossano JW, et al. An underrecognized benefit of cardiopulmonary resuscitation: organ transplantation. Crit Care Med 2013;41:2794-2799.

Patel MS, Niemann CU, Sally MB, et al. The Impact of Hydroxyethyl Starch Use in Deceased Organ Donors on the Development of Delayed Graft Function in Kidney Transplant Recipients: a Propensity-Adjusted Analysis. Am J Transplant 2015;15:2152-2158.

Polderman KH. Induced hypothermia and fever control for prevention and treatment of neurological injuries. Lancet 2008;371:1955-1969.

Polderman KH. Mechanisms of action, physiological effects, and complications of hypothermia. Crit Care Med 2009; 37(7 Suppl):S186-S202.

Schnuelle P, Gottmann U, Hoeger S, et al. Effects of donor pretreatment with dopamine on graft function after kidney transplantation: a randomized controlled trial. JAMA 2009; 302:1067-1075. 
Serrano OK, Vock DM, Chinnakotla $\mathrm{S}$, et al. The relationships between cold ischemia time, kidney transplant length of stay, and transplant-related costs. Transplantation 2018;103:401411.

Tapiawala SN, Tinckam KJ, Cardella CJ, et al. Delayed graft function and the risk for death with a functioning graft. J Am Soc Nephrol 2010;21:153-161.

West S, Soar J, Callaway CW. The viability of transplanting organs from donors who underwent cardiopulmonary resuscitation: a systematic review. Resuscitation 2016;108:27-33.

Yarlagadda SG, Coca SG, Formica RN, Jr., et al. Association between delayed graft function and allograft and patient survival: a systematic review and meta-analysis. Nephrol Dial Transplant 2009;24:1039-1047.
Yenari MA, Han HS. Neuroprotective mechanisms of hypothermia in brain ischaemia. Nat Rev Neurosci 2012;13:267-278.

Zens TJ, Danobeitia JS, Leverson G, et al. The impact of kidney donor profile index on delayed graft function and transplant outcomes: a single-center analysis. Clin Transplant 2018;2: e13190.

Address correspondence to:

Darren J. Malinoski, MD, FACS

VA Portland Health Care System (VAPORHCS)

P.O. Box 1034/P3SURG

Portland, OR 97207

E-mail: malinosk@ohsu.edu 